\title{
Podoplanin Expression in Canine Melanoma
}

\author{
Satoshi Ogasawara, ${ }^{1, *}$ Ryusuke Honma, ${ }^{1, *}$ Mika K. Kaneko,, Yuki Fujii, \\ Yumiko Kagawa, Satoru Konnai, and Yukinari Kato ${ }^{1}$
}

A type I transmembrane protein, podoplanin (PDPN), is expressed in several normal cells such as lymphatic endothelial cells or pulmonary type I alveolar cells. We recently demonstrated that anticanine PDPN monoclonal antibody (mAb), PMab-38, recognizes canine PDPN of squamous cell carcinomas, but does not react with lymphatic endothelial cells. Herein, we investigated whether PMab-38 reacts with canine melanoma. PMab-38 reacted with 90\% of melanoma cells (9/10 cases) using immunohistochemistry. Of interest, PMab-38 stained the lymphatic endothelial cells and cancer-associated fibroblasts in melanoma tissues, although it did not stain any lymphatic endothelial cells in normal tissues. PMab-38 could be useful for uncovering the function of PDPN in canine melanomas.

Keywords: canine podoplanin, melanoma, monoclonal antibody, immunohistochemistry

A TYPE I TRANSMEMBRANE SIALOGLYCOPROTEIN, pOdoplanin (PDPN), is also known as gp40/T1 $\alpha /$ Aggrus. ${ }^{(1-5)}$ PDPN is expressed in normal cells including renal podocytes, pulmonary type I alveolar cells, and lymphatic endothelial cells. ${ }^{(4)}$ PDPN activates platelet aggregation by binding to Ctype lectin-like receptor-2 (CLEC-2) on platelets, ${ }^{(6,7)}$ and the PDPN-CLEC-2 interaction facilitates blood/lymphatic vessel separation. ${ }^{(8)}$ The expression of human PDPN was reported in many tumors, including oral cancers, ${ }^{(9)}$ malignant brain tumors, ${ }^{(10-12)}$ lung cancers, ${ }^{(13)}$ esophageal cancers, ${ }^{(14)}$ malignant mesotheliomas, ${ }^{(15)}$ testicular tumors, ${ }^{(16)}$ and osteosarcomas. ${ }^{(17)}$ PDPN expression is also associated with cancer metastasis and malignant progression. ${ }^{(6,10)}$

We previously developed an anticanine PDPN monoclonal antibody (mAb), PMab-38, ${ }^{(18)}$ which is useful for immunohistochemistry (IHC), flow cytometry, and Western blotting. Recently, we demonstrated that PMab-38 can recognize PDPN of canine squamous cell carcinomas using IHC. ${ }^{(19)}$ Tumor cells in 15 out of 18 canine squamous cell carcinomas $(83 \%)$ were stained by PMab-38 in IHC. Cancer-associated fibroblasts in 14 out of 18 cases $(78 \%)$ were detected by PMab-38. In this study, we investigated whether canine melanoma was stained by PMab-38 because mouse PDPN expression and human PDPN expression were observed in melanomas. ${ }^{(20,21)}$ Watanabe et al. reported that high expression of mouse PDPN is associated with the metastatic ability in metastatic variants of B16 melanomas. ${ }^{(20)}$
We stained 10 canine oral melanomas, which are the most frequent oral cancers in dog. ${ }^{(22)}$ As depicted in Figures 1 and 2A, melanoma cells were stained by PMab-38 in 9 of 10 cases. Although melanoma cells were not stained by PMab-38 in case 9 (Fig. 1), cancer-associated fibroblasts were recognized by PMab-38 (Fig. 2B). Both melanoma cells and cancer-associated fibroblasts were stained in case 5 (Fig. 1). Kan et al. reported that PDPN expression in cancer-associated fibroblasts correlates with aggressive behavior in human melanoma, ${ }^{(21)}$ indicating that PDPN in cancer-associated fibroblasts of melanoma tissues might serve as a useful prognostic factor not only in human melanoma but also in canine melanoma. As shown in Figure 2C, PDPN of lymphatic endothelial cells was detected by PMab-38 in melanoma tissues, although lymphatic endothelial cells in normal tissues ${ }^{(18)}$ or squamous cell carcinomas ${ }^{(19)}$ were not stained by PMab-38, indicating that PDPN expression might be upregulated in melanoma, or post-translational modification of canine PDPN might be different between squamous cell carcinomas and melanomas.

We recently reported that the PMab-38 epitope is far from the platelet aggregation-stimulating domain of PDPN. ${ }^{(19)}$ However, we have not clarified whether the epitope of PMab38 includes the post-translational modification. In the near future, we should determine the PMab-38 epitope using alanine scanning or glycan-deficient cells and uncover the difference of PDPN expression in several tissues.

\footnotetext{
${ }^{1}$ Department of Regional Innovation, Tohoku University Graduate School of Medicine, Miyagi, Japan.

${ }^{2}$ North Lab, Hokkaido, Japan.

${ }^{3}$ Department of Disease Control, Graduate School of Veterinary Medicine, Hokkaido University, Hokkaido, Japan.

*These authors contributed equally to this work.
} 


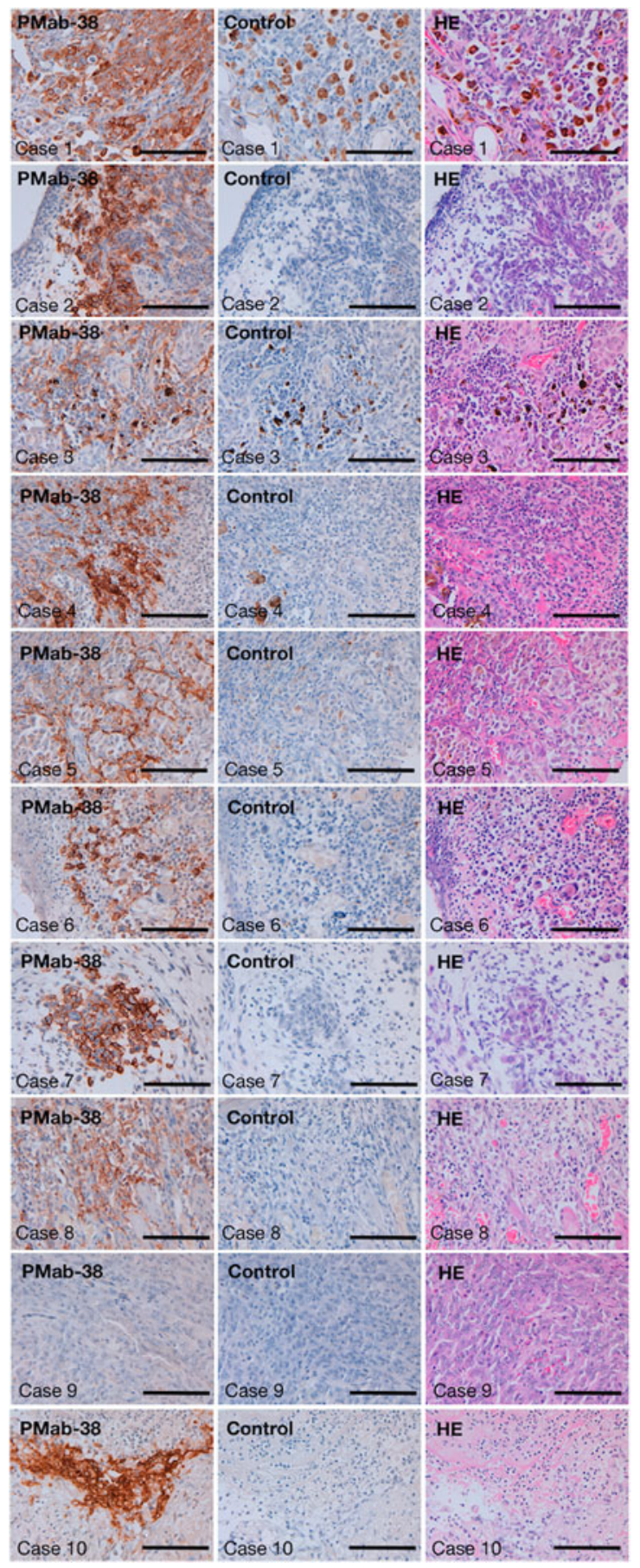

FIG. 1. Immunohistochemical analysis against canine melanoma using PMab-38. Canine melanomas (10 cases) were obtained from North Lab (Hokkaido, Japan). Four micrometers thick histologic sections were deparaffinized in xylene, rehydrated, and autoclaved in citrate buffer ( $\mathrm{pH} \mathrm{6.0;}$ Dako, Glostrup, Denmark) for 20 minutes. Sections were incubated with $10 \mu \mathrm{g} / \mathrm{mL}$ of PMab-38 overnight at $4^{\circ} \mathrm{C}$ followed by treatment with Envision+ kit for 30 minutes (Dako). As a control, blocking buffer was used in this study. Color was developed using 3,3-diaminobenzidine tetrahydrochloride (Dako) for 2 minutes, after which the sections were counterstained with hematoxylin (Wako Pure Chemical Industries Ltd., Osaka, Japan). H\&E staining was also performed. Scale bar: $100 \mu \mathrm{m}$. mAb, monoclonal antibody; PDPN, podoplanin.
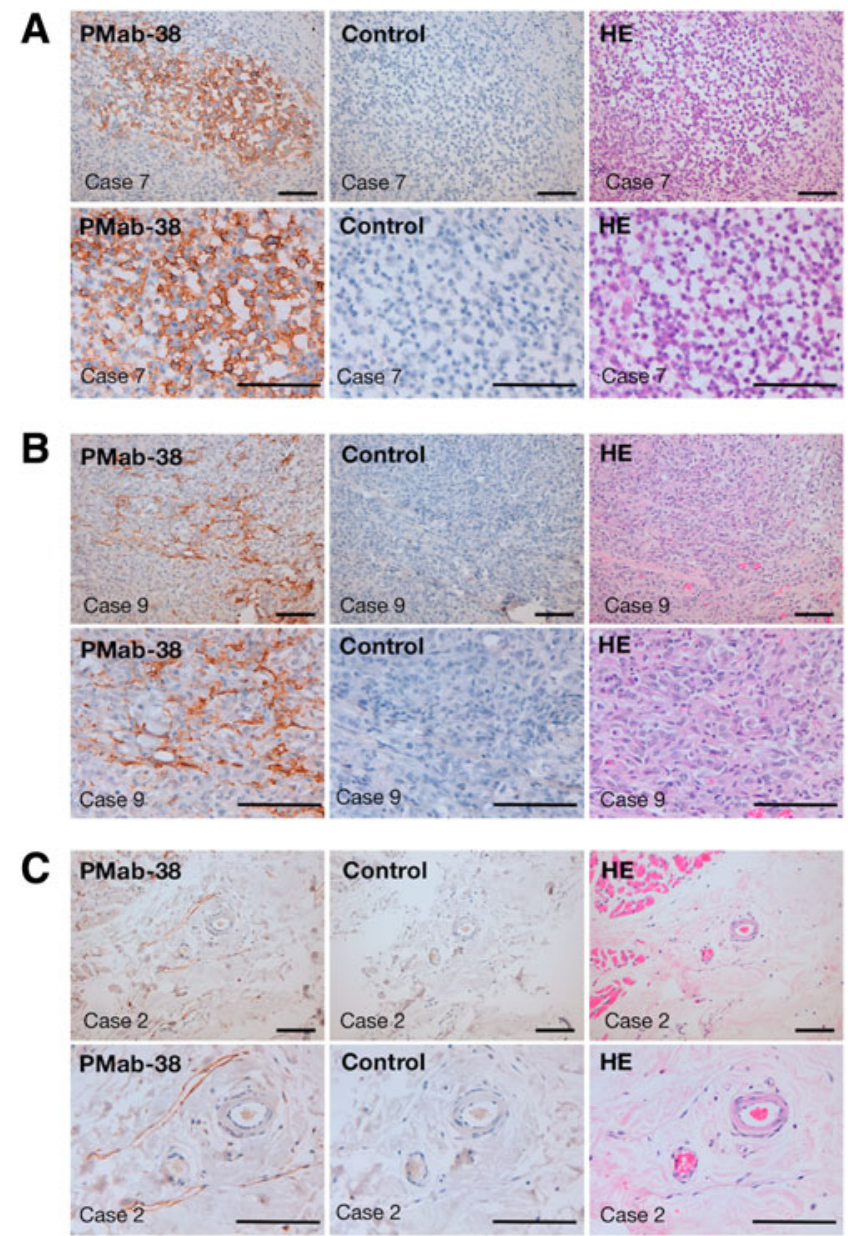

FIG. 2. PMab-38 reacted with PDPN of melanoma cells, cancer-associated fibroblasts, and lymphatic endothelial cells. Sections of melanomas (A, case $7 ; \mathbf{B}$, case $9 ; \mathbf{C}$, case 2 ) were incubated with $10 \mu \mathrm{g} / \mathrm{mL}$ of PMab-38 overnight at $4^{\circ} \mathrm{C}$ followed by treatment with Envision+ kit for 30 minutes (Dako). As a control, blocking buffer was used. Color was developed using 3,3-diaminobenzidine tetrahydrochloride (Dako) for 2 minutes, after which the sections were counterstained with hematoxylin (Wako). H\&E staining was also performed. Scale bar: $100 \mu \mathrm{m}$. (A) staining of melanoma cells by PMab38, (B) staining of cancer-associated fibroblasts by PMab-38, and (C) staining of lymphatic endothelial cells by PMab-38.

\section{Acknowledgments}

We thank Takuro Nakamura, Noriko Saidoh, and Kanae Yoshida for their excellent technical assistance. This work was supported, in part, by the Regional Innovation Strategy Support Program from the Ministry of Education, Culture, Sports, Science and Technology (MEXT) of Japan (Y.K.), by JSPS KAKENHI Grant Numbers 26440019 (M.K.K.) and $16 \mathrm{~K} 10748$ (Y.K.), by the Platform for Drug Discovery, Informatics, and Structural Life Science (PDIS) from Japan Agency for Medical Research and Development, AMED (Y.K.), by Project for utilizing glycans in the development of innovative drug discovery technologies from AMED (Y.K.), and by the Basic Science and Platform Technology Program for Innovative Biological Medicine from AMED (Y.K.). This work was performed, in part, under the Cooperative Research Program of Institute for Protein Research, Osaka University, 
CR-16-05 and by the Grant for Joint Research Project of the Institute of Medical Science, the University of Tokyo.

\section{Author Disclosure Statement}

No competing financial interests exist.

\section{References}

1. Zimmer G, Klenk HD, and Herrler G: Identification of a 40$\mathrm{kDa}$ cell surface sialoglycoprotein with the characteristics of a major influenza $\mathrm{C}$ virus receptor in a Madin-Darby canine kidney cell line. J Biol Chem 1995;270:17815-17822.

2. Kato Y, Fujita N, Kunita A, Sato S, Kaneko M, Osawa M, and Tsuruo T: Molecular identification of Aggrus/T1alpha as a platelet aggregation-inducing factor expressed in colorectal tumors. J Biol Chem 2003;278:51599-51605.

3. Kaneko MK, Kato Y, Kitano T, and Osawa M: Conservation of a platelet activating domain of Aggrus/podoplanin as a platelet aggregation-inducing factor. Gene 2006;378:52-57.

4. Breiteneder-Geleff S, Matsui K, Soleiman A, Meraner P, Poczewski H, Kalt R, Schaffner G, and Kerjaschki D: Podoplanin, novel 43-kd membrane protein of glomerular epithelial cells, is down-regulated in puromycin nephrosis. Am J Pathol 1997;151:1141-1152.

5. Martin-Villar E, Scholl FG, Gamallo C, Yurrita MM, Munoz-Guerra M, Cruces J, and Quintanilla M: Characterization of human PA2.26 antigen (T1alpha-2, podoplanin), a small membrane mucin induced in oral squamous cell carcinomas. Int J Cancer 2005;113:899-910.

6. Kato Y, Kaneko MK, Kunita A, Ito H, Kameyama A, Ogasawara S, Matsuura N, Hasegawa Y, Suzuki-Inoue K, Inoue O, Ozaki Y, and Narimatsu H: Molecular analysis of the pathophysiological binding of the platelet aggregationinducing factor podoplanin to the C-type lectin-like receptor CLEC-2. Cancer Sci 2008;99:54-61.

7. Kaneko MK, Kunita A, Abe S, Tsujimoto Y, Fukayama M, Goto K, Sawa Y, Nishioka Y, and Kato Y: Chimeric antipodoplanin antibody suppresses tumor metastasis through neutralization and antibody-dependent cellular cytotoxicity. Cancer Sci 2012;103:1913-1919.

8. Bertozzi CC, Schmaier AA, Mericko P, Hess PR, Zou Z, Chen M, Chen CY, Xu B, Lu MM, Zhou D, Sebzda E, Santore MT, Merianos DJ, Stadtfeld M, Flake AW, Graf T, Skoda R, Maltzman JS, Koretzky GA, and Kahn ML: Platelets regulate lymphatic vascular development through CLEC-2-SLP-76 signaling. Blood 2010;116:661-670.

9. Ochoa-Alvarez JA, Krishnan H, Pastorino JG, Nevel E, Kephart D, Lee JJ, Retzbach EP, Shen Y, Fatahzadeh M, Baredes S, Kalyoussef E, Honma M, Adelson ME, Kaneko MK, Kato Y, Young MA, Deluca-Rapone L, Shienbaum AJ, Yin K, Jensen LD, and Goldberg GS: Antibody and lectin target podoplanin to inhibit oral squamous carcinoma cell migration and viability by distinct mechanisms. Oncotarget 2015;6:9045-9060.

10. Mishima K, Kato Y, Kaneko MK, Nishikawa R, Hirose T, and Matsutani $\mathrm{M}$ : Increased expression of podoplanin in malignant astrocytic tumors as a novel molecular marker of malignant progression. Acta Neuropathol (Berl) 2006;111: 483-488.

11. Kato Y, Vaidyanathan G, Kaneko MK, Mishima K, Srivastava N, Chandramohan V, Pegram C, Keir ST, Kuan CT, Bigner DD, and Zalutsky MR: Evaluation of anti-podoplanin rat monoclonal antibody NZ-1 for targeting malignant gliomas. Nucl Med Biol 2010;37:785-794.
12. Kato Y, and Kaneko MK: A cancer-specific monoclonal antibody recognizes the aberrantly glycosylated podoplanin. Sci Rep 2014;4:5924.

13. Kato Y, Kaneko M, Sata M, Fujita N, Tsuruo T, and Osawa M: Enhanced expression of Aggrus (T1alpha/podoplanin), a platelet-aggregation-inducing factor in lung squamous cell carcinoma. Tumor Biol 2005;26:195-200.

14. Schoppmann SF, Jesch B, Riegler MF, Maroske F, Schwameis K, Jomrich G, and Birner P: Podoplanin expressing cancer associated fibroblasts are associated with unfavourable prognosis in adenocarcinoma of the esophagus. Clin Exp Metastasis 2013;30:441-446.

15. Abe S, Morita Y, Kaneko MK, Hanibuchi M, Tsujimoto Y, Goto H, Kakiuchi S, Aono Y, Huang J, Sato S, Kishuku M, Taniguchi Y, Azuma M, Kawazoe K, Sekido Y, Yano S, Akiyama S, Sone S, Minakuchi K, Kato Y, and Nishioka Y: A novel targeting therapy of malignant mesothelioma using anti-podoplanin antibody. J Immunol 2013; 190:6239-6249.

16. Kato Y, Sasagawa I, Kaneko M, Osawa M, Fujita N, and Tsuruo T: Aggrus: A diagnostic marker that distinguishes seminoma from embryonal carcinoma in testicular germ cell tumors. Oncogene 2004;23:8552-8556.

17. Kaneko MK, Oki H, Ogasawara S, Takagi M, and Kato Y: Anti-podoplanin monoclonal antibody LpMab-7 detects metastatic legions of osteosarcoma. Monoclon Antib Immunodiagn Immunother 2015;34:154-161.

18. Honma R, Kaneko MK, Ogasawara S, Fujii Y, Konnai S, Takagi M, and Kato Y: Specific detection of dog podoplanin expressed in renal glomerulus by a novel monoclonal antibody PMab-38 in immunohistochemistry. Monoclon Antib Immunodiagn Immunother 2016;35:212-216.

19. Kaneko MK, Honma R, Ogasawara S, Fujii Y, Nakamura T, Saidoh N, Takagi M, Kagawa Y, Konnai S, and Kato Y: PMab-38 recognizes canine podoplanin of squamous cell carcinomas. Monoclon Antib Immunodiagn Immunother 2016;35:263-266.

20. Watanabe M, Sugimoto $\mathrm{Y}$, and Tsuruo T: Expression of a $\mathrm{Mr} 41,000$ glycoprotein associated with thrombinindependent platelet aggregation in high metastatic variants of murine B16 melanoma. Cancer Res 1990;50:6657-6662.

21. Kan S, Konishi E, Arita T, Ikemoto C, Takenaka H, Yanagisawa $\mathrm{A}$, Katoh N, and Asai J: Podoplanin expression in cancer-associated fibroblasts predicts aggressive behavior in melanoma. J Cutan Pathol 2014;41:561-567.

22. Maekawa N, Konnai S, Okagawa T, Nishimori A, Ikebuchi R, Izumi Y, Takagi S, Kagawa Y, Nakajima C, Suzuki Y, Kato Y, Murata S, and Ohashi K: Immunohistochemical analysis of PD-L1 expression in canine malignant cancers and PD-1 expression on lymphocytes in canine oral melanoma. PLoS One 2016;11:e0157176.

Address correspondence to: Dr. Yukinari Kato Department of Regional Innovation Tohoku University Graduate School of Medicine 2-1 Seiryo-machi, Aoba-ku Sendai, Miyagi 980-8575 Japan

E-mail: yukinari-k@bea.hi-ho.ne.jp; yukinarikato@med.tohoku.ac.jp

Received: September 9, 2016 Accepted: October 21, 2016 